\title{
ANÁLISES QUÍMICA E BROMATOLÓGICAS DE SEMENTES E DE ÓLEO FIXO DE MELANCIA (CITRULLUS LANATUS, CUCURBITACEAE)
}

\author{
CHEMICAL AND BROMATOLOGICAL ANALYZES OF SEEDS AND FIXED OIL OF \\ WATERMELON (CITRULLUS LANATUS, CUCURBITACEAE)
}

\author{
Angelica Victoria de Campos SOUZA ${ }^{1}$; Beatriz Sponholz OLIVEIRA ${ }^{1}$; Giovanna \\ Beatriz Sari HEY'; Samuel Henrique WITT'1; Maria Eugenia BALBI²; Francinete \\ Ramos CAMPOS ${ }^{2}$
}

1 - Discente do Curso de Farmácia, Universidade Federal do Paraná (UFPR).

2 - Professora do Departamento de Farmácia, Universidade Federal do Paraná (UFPR).

Autor para correspondência: bromatologia.ufpr@gmail.com

\section{RESUMO:}

A semente de melancia apresenta importância econômica para o Brasil, sendo produzida em grande escala anualmente, entretanto, o que é usado se resume à polpa, sendo a semente descartada ou usada apenas em chás. Visando evitar desperdício e suplementar a alimentação, esse estudo traz informações acerca das propriedades nutricionais e químicas da semente de melancia e de seu óleo fixo. Os processamentos da semente resultaram em importantes descobertas como uma alta taxa de fibras $(40,47 \%)$ e proteína $(17,26 \%)$ na farinha, além de grande concentração de ácido linoleico, que é conhecido por reduzir a massa corporal. Ainda, sugere-se que novas análises acerca do óleo sejam feitas. Palavras chave: Semente de melancia; Citrullus lanatus, Ácido linoleico; $\mathrm{RMN}-\mathrm{H}^{1}$; Farinha.

\section{ABSTRACT:}

The watermelon seed has economic contribution to Brazil, being produces in large scale every year, however, what is viable resumes to its pulp where the seed is discarded or used only to make tea. Aiming to avoid wasting and to supplementary feeding, the present study brings information about watermelon seed nutritional and chemical facts and its fixed oil. The processing of seed resulted in important findings like the high fibers $(40,47 \%)$ and protein $(17,26 \%)$ rate in the flour, besides a huge concentration of linoleic acid in the oil, which is known for reducing corporal mass. Notwithstanding these limitations, the study suggests that further analyses may be done.

KEYWORDS: watermelon sed; Citrullus lanatus; Linoleic acid; NMR-H1; flour.

\section{INTRODUÇÃO}

A melancia (Citrullus lanatus) é uma planta com origem na África Equatorial, sendo, no Brasil, uma das olerícolas mais importantes em relação à produção e comercialização, ficando somente atrás de tomate, batata e cebola. Além disso, é a planta da família Cucurbitaceae mais produzida no mundo. Pertencem a esta família abóbora, melão, abobrinha, pepino, etc, ou seja, plantas de haste rastejante com gavinhas de sustentação 
(ANDRADE, et. al., 2007). De acordo com dados da Produção Agrícola Municipal (PAM) do Instituto Brasileiro de Geografia e Estatística (IBGE), foram produzidas mais de 2 milhões de toneladas de melancia no ano de 2017 (IBGE, 2017).

A polpa da melancia é ricamente composta por umidade (90,7\%), e $100 \mathrm{~g}$ do produto oferecem $33 \mathrm{kcal}, 10 \mathrm{mg}$ de magnésio, $8 \mathrm{mg}$ de cálcio, $6,1 \mathrm{mg}$ de vitamina $\mathrm{C}$, além de quantidades menores de equivalente de retinol (TACO, 2011). Sua coloração vermelha se dá pela presença de licopeno, o qual, por sua vez, é fonte dos aminoácidos arginina e citrulina.

A demanda por alimentos saudáveis cresce mundialmente. Refeições balanceadas permitem a prevenção de doenças cardiovasculares, desnutrição, diabetes, obesidade, dentre outros (GUTKOSKI, et. al., 2007). Entretanto, muitos produtos de origem vegetal in natura são desperdiçados em seus processos de preparação para o transporte, venda e também no ambiente doméstico. No caso da melancia, sementes e casca acabam por tornar-se um fardo para consumidor que pretende alimentar-se somente da fruta. Subprodutos de frutas e hortaliças (talos, cascas, etc.) podem apresentar quantidades significativas de fibras e outros componentes essenciais à nutrição humana (PRIM, et. al., 2003). As semestes da melancia, por exemplo, podem ser utilizadas no formato de chás ou para a fabricação de farinhas, embora esta última opção não seja muito comum.

Existem estudos que propõe a utilização da entrecasca da melancia para produção de farinha por conta do seu alto teor de fibras (GUIMARÃES, 2008), e outros estudos onde o óleo de semete de frutas são analisados, comprovando a capacidade antioxidante desse tipo de óleos e seu potencial uso em alimentos e cosméticos (DENG, et. al., 2018). Não existem, porém, muitos estudos tratando do uso para fins culinários, nutricionais e/ou terapêuticos das sementes de melancia, tanto na forma de farinha como do óleo fixo extraído da semente. Dessa forma, o objetivo deste trabalho foi analisar quantitativamente e nutricionalmente as sementes de melancia e propor maneiras de utilizá-las na alimentação, além de analisar quimicamente seu óleo fixo.

\section{MATERIAL E MÉTODOS}

Neste estudo foram utilizadas sementes de melancia obtidas a partir de 2 melancias, cada uma pesando $11,04 \mathrm{~kg}$ e $13,03 \mathrm{~kg}$. As frutas foram adquiridas em supermercado da cidade de Curitiba - PR em agosto de 2018. 


\subsection{Análise sensorial e bromatológica}

A amostra de sementes de melancia foi extraída mecanicamente das frutas, com auxílio de faca, e mantida em refrigeração até o momento da análise sensorial. Foi feito o quarteamento da amostra em bancada descontaminada com álcool etílico $70 \%$. Após esta etapa, as sementes foram submetidas a um procedimento de moagem por aproximadamente 10 minutos em liquidificador caseiro e posterior secagem, a 50ㄷ, em estufa. Após a secagem, a amostra passou a ser chamado de farinha.

Esta farinha passou pelas seguintes análises de composição química e nutricional: determinação de umidade pelo método de voláteis a $105^{\circ} \mathrm{C}$ (IAL, 2008), determinação de nitrogênio total pelo método de Micro Kjeldahl (AOAC, 960.52, 1995; MODIFICADO) utilizando o fator de conversão de proteínas de 5,75 conforme previsto no Regulamento Técnico sobre Rotulagem Nutricional de Alimentos Embalados da Resolução da Diretoria Colegiada (RDC) 360 (BRASIL, 2003), determinação da fração de extrato etéreo (IAL, 2008), determinação de fibras (AOAC, 1970) e determinação de minerais (IAL, 2008). A determinação de carboidratos foi feita por diferença, de acordo com a equação abaixo:

$$
C \%=100 \%-(U \%+P \%+L \%+M \%+F \%)
$$

EQUAÇÃO 1: Determinação de carboidratos (C). U: umidade; P: proteínas; L: lipídeos; M: minerais; F: fibras.

As análises foram realizadas no Laboratório de Bromatologia do Departamento de Farmácia da Universidade Federal do Paraná (UFPR), Campus Jardim Botânico, Curitiba, Paraná, Brasil.

\subsection{Análise espectroscópica}

O espectro do óleo fixo oriundo da extração etérea (IAL, 2008) foi obtido quantitativamente em $\mathrm{CDCl} 3$ a $30^{\circ} \mathrm{C}$ em um espectrômetro de RMN (Ressonância Magnética Nuclear) Bruker Avance III 600, operando a 14,1 Tesla, observando o ${ }^{1} \mathrm{H}$ a $600,13 \mathrm{MHz}$, equipado com uma sonda de detecção a $5 \mathrm{~mm}$ quadri nuclear inversa com gradiente $z$. Todos os deslocamentos químicos de RMN de ${ }^{1} \mathrm{H} \delta$ são dados em ppm em relação ao sinal TMS a 0,00 ppm como referência interna, e as constantes de acoplamento (J) em Hz. (BARISON, et. al., 2010). Esta análise foi feita em colaboração com o 
Departamento de Química da Universidade Federal do Paraná sob a coordenação do Prof. Andersson Barison.

\section{RESULTADOS E DISCUSSÃO}

\subsection{Análise sensorial}

No quarteamento da amostra, foi possível verificar resquícios de fruta nas sementes. O odor era o característico da polpa da melancia e a cor das sementes variava em tonalidades de marrom claro. Praticamente nenhuma semente estava em processo de germinação.

\subsection{Composição química}

Os resultados obtidos após as análises bromatológicas estão expostos na TABELA 1. Podemos notar valores semelhantes aos já descritos na literatura (SELF NUTRITION, 2018).

TABELA 1: Composição química da semente in natura e da farinha de semente de melancia (Citrullus lanatus). DP: desvio padrão.

\begin{tabular}{lcc}
\hline \multirow{2}{*}{ Determinação } & In natura & Farinha \\
\cline { 2 - 3 } & Composição (\%) [DP] & Composição (\%) [DP] \\
\hline Umidade & $55,29[2,97]$ & $8,60[0,21]$ \\
Pipídeos & $11,06[0,12]$ & $24,75[0,27]$ \\
Carboteínas* & $7,72[0,64]$ & $17,26[1,44]$ \\
Fibras & 9,46 & 5,48 \\
Minerais & $15,31[0,69]$ & $40,47[1,84]$ \\
kcal (100g) & $1,16 \pm[0,24]$ & $3,46 \pm[0,72]$ \\
\hline
\end{tabular}

*Utilizando o fator de conversão 5,75 conforme Regulamento Técnico sobre Rotulagem Nutricional de Alimentos Embalados da RDC 360/2003.

A umidade é um fator importante a ser levado em consideração, uma vez que tem relação direta com a deterioração da matéria por microorganismos. Pode-se fazer, também, 
uma associação dela com a 'diluição' dos compostos presentes na semente de melancia, pois, os elementos quantificados estão mais concentrados e em maior quantidade na farinha do que na semente, com exceção dos carboidratos. Isso pode-se explicar pelo fato da determinação dos carboidratos ter sido feita por diferença e o cálculo envolver reduzir de $100 \%$ - todas a médias das outras quantificações, e consequentemente, pelo fato da farinha apresentar médias maiores dos outros compostos determinados, acaba por ter o nível de carboidratos reduzido.

As características físico-químicas das fibras promovem efeitos locais e sistêmicos no organismo humano. As diferenças quanto à capacidade de retenção de água, viscosidade, fermentação, adsorção, entre outras, são responsáveis por implicações metabólicas (efeitos sistêmicos), bem como no trato gastrointestinal (efeitos locais) (BUTTRISS, et. al., 2008). E é, também, por isso que a farinha de semente de melancia chamou atenção, devido à grande quantidade de fibras e em como isso pode impactar na nutrição de quem a consumir.

\subsection{Análise espectroscópica}

A obtenção de óleo da semente de melancia teve rendimento aproximado de $25 \%$. O óleo foi analisado por Ressonância Magnética Nuclear (RMN) de hidrogênio. A análise identificou sinais de triacilglicerol (FIGURA 1) contendo ácidos oléico, linoléico e ácidos graxos saturados. Por conseguinte, o teor relativo de cada ácido graxo esterificado para o radical glicerol, revelando que as sementes oleaginosas são compostas por ácidos graxos saturados sendo palmítico e esteárico (23,3\%), e insaturados oléico $(19,4 \%)$ e linoleico (89,9\%).

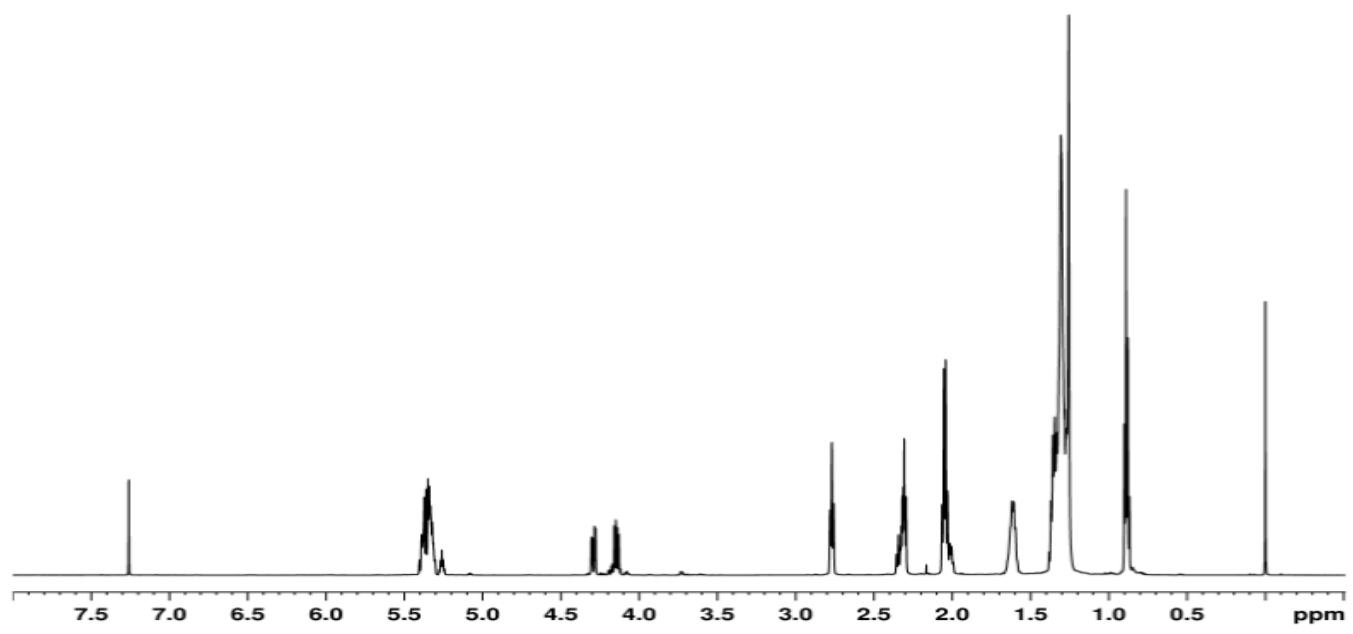

FIGURA 1: Espectro de RMN de 1H do óleo das sementes de melancia. Fonte: OS AUTORES, 2018 
As gorduras e óleos são nutrientes essenciais na alimentação humana, além de serem excelentes fontes de energia. Alguns ácidos graxos são essenciais pela impossibilidade dos animais sintetizarem estes lipídios a partir de precursores simples (MARTIN, et. al., 2006). O ácido linoleico, encontrado no teor de $89,9 \%$ no $\mathrm{RMN}^{1} \mathrm{H}$, é um ácido graxo poliinsaturado também conhecido como ômega-6 pelo fato de possuir sua primeira insaturação no sexto carbono enumerado a partir do grupo metil terminal. $\mathrm{O}$ ácido linoleico conjugado é uma outra forma de ômega-6 (uma mistura de isômeros de ácidos linoleicos com duplas ligações conjugadas) (GAULIER, et. al., 2004) que possui benefícios para a saúde, incluindo redução da massa corporal (WHIGHAM, et. al., 2007).

O ácido oléico (que constitui 19,4\% do óleo fixo da semente de melancia), chamado de ômega-9, é também um ácido graxo com 18 carbonos. Sua concentração é alta nos óleos de soja, oliva, palma e em animais marinhos. Seu consumo está relacionado com diminuição dos níveis de VLDL (very low density lipoprotein) e de colesterol, além de dietas ricas em ômega-9 oferecerem vantagens a pacientes com diabetes tipos 1 e 2 por sua capacidade antioxidante, conforme demonstrado em um estudo de meta-análise (GARG, et. al., 1998).

O teor de ácido linoleico no óleo da semente de melancia é maior que em outros óleos vegetais (oliva, milho, girassol, soja, algodão e amendoim). Já a porcentagem de ácido oleico é menor em todos os óleos vegetais citados anteriormente. O teor de ácidos graxos saturados é semelhante a maioria dos óleos. (FONSECA, et. Al., 1974).

\subsection{INFORMAÇÃO NUTRICIONAL}

TABELA 2: Informação nutricional porção da semente in natura e da farinha da semente de melancia, em conformação com a legislação.

\begin{tabular}{lcccc}
\hline & \multicolumn{2}{c}{$\begin{array}{c}\text { SEMENTE } \\
\text { Porção de 12 } \mathrm{g}\end{array}$} & & \multicolumn{2}{c}{$\begin{array}{c}\text { FARINHA } \\
\text { Porção de 12 } \mathrm{g} \\
\text { (duas colheres de sopa) }\end{array}$} \\
\cline { 2 - 5 } & g da porção & VD\%* & g da porção & VD\%* \\
\hline Valor energético & $20,19 \mathrm{kcal} / 84,53 \mathrm{~kJ}$ & $1 \%$ & $37,54 \mathrm{kcal} / 157,59 \mathrm{~kJ}$ & $1,87 \%$ \\
\hline Carboidratos & $1,14 \mathrm{~g}$ & $0,38 \%$ & $0,66 \mathrm{~g}$ & $0,22 \%$ \\
\hline Proteínas & $0,93 \mathrm{~g}$ & $1,23 \%$ & $2,07 \mathrm{~g}$ & $2,76 \%$ \\
\hline Gorduras totais & $1,33 \mathrm{~g}$ & $2,41 \%$ & $2,97 \mathrm{~g}$ & $5,40 \%$ \\
\hline Fibra Alimentar & $1,84 \mathrm{~g}$ & $7,35 \%$ & $4,85 \mathrm{~g}$ & $19,42 \%$ \\
\hline
\end{tabular}

*Valores diários de Referência com base em uma dieta de 2.000 kcal ou 8.400 kJ. 
O conhecimento da composição nutricional da semente de melancia é muito importante para que seja possível fazer um estudo sobre como empregar essa semente na alimentação. A TABELA 2 traz informações acerca de aspectos nutricionais da semente in natura e da farinha, com os valores diários em porcentagem.

É válido destacar, nesta tabela, a alta taxa de fibras presentes em uma amostra de 12 gramas que, quando comparada com a farinha de aveia desengordurada - que em 100 $\mathrm{g}$ apresenta cerca de 9,69 $\mathrm{g}$ de fibras totais (DANIEL, et. al., 2006) - pode-se ver a clara superioridade da farinha de semente de melancia. Convertendo-se para a mesma porção, a farinha de aveia desengordurada contribui em apenas $4,65 \%$ nos valores diários de fibras, enquanto que a farinha de semente de melancia agrega em 19,44\%.

Ainda convém comparar a farinha de semente de melancia com a farinha de chia, e os valores nutricionais de ambas são semelhantes, entretanto, a segunda apresentou-se mais gordurosa, proteica, com maior quantidade de fibras e concentração menor de minerais - 2,33\% de cinzas em $100 \mathrm{~g}$ (PUIG e HAROS, 2011). Logo, apesar de serem quase equivalentes, a farinha de semente de chia ostenta um alto custo, podendo dificultar sua adição à dieta e ao consumo diário, enquanto que, a farinha de semente de melancia ainda não está presente no mercado para poder se fazer uma comparação efetiva de custobenefício entre as duas.

Os outros valores comparados mantiveram a proporção entre os dados tidos da composição química da farinha e da semente, estando a semente como mais rica em carboidratos e sendo menos energética, e a farinha com maiores quantidades de fibras, proteínas e gorduras, além de apresentar-se mais calórica.

\section{CONCLUSÃO}

O presente estudo demonstrou que a farinha da semente de melancia é uma ótima fonte nutritiva, possuindo alto valor proteico e de fibras, além de ser pouco calórica e com um teor baixo de carboidrato. A análise de $\mathrm{RMN}^{-1} \mathrm{H}$ realizada no óleo fixo da semente de melancia demonstrou a presença de ácidos graxos insaturados (linoleico e oleico) importantes na nutrição humana.

Pelo fato de ser muito produzida e consumida no Brasil, sugerimos que mais estudos com os subprodutos da melancia sejam feitos para que possamos começar a incluílos em nossa dieta. No caso da farinha da semente, podem ser feitos estudos acerca da incorporação desta em produtos como bolos, tortas e barras de cereal. Para o óleo fixo da 
semente, sugerimos a realização de testes para a avaliação das propriedades terapêuticas deste óleo, como capacidade antioxidante e poder antimicrobiano.

\section{REFERÊNCIAS}

ANDRADE, A. S. A cultura de melancia. 2. ed. Brasília, DF: Embrapa Informação Tecnológica, 2007. 85 p. Disponível em: <https://ainfo.cnptia.embrapa.br/digital/bitstream/ item/11919/2/00081320.pdf>. Acesso em: 16 set. 2018.

A.O.A.C. ASSOCIATION OF OFFICIAL ANALYTICAL CHEMISTS. Official Methods of Analysis of Association of Official Analytical Chemists. 16th ed. Washington D.C. 1995.

BARISON, A.; SILVA, C. W.; CAMPOS, F. R.; SIMONELLI, F.; LENZ, C. A.; FERREIRA, A. G. Simple methodology for the determination of fatty acid composition in edible oils through ${ }^{1} \mathrm{H}$ NMR spectroscopy. Magnetic resonance in chemistry: MRC, v. 48, n. 8, p. 642-650, 2010. Disponível em: <https://www.ncbi.nlm.nih.gov/pubmed/20589730>. Acesso em: 08 nov. 2018.

BERNAUD, F. S. R.; RODRIGUES, T. C. Fibra alimentar - Ingestão adequada e efeitos sobre a saúde do metabolismo. 2012. 9 f. Dissertação (Fibra alimentar - Ingestão adequada e efeitos sobre a saúde do metabolismo. Programa de Pós-Graduação em Ciências Médicas: Endocrinologia) - Universidade Federal do Rio Grande do Sul, UFRGS, Porto Alegre, 2013. 57/6. Disponível em: <http://www.scielo.br/pdf/abem/v57n6/01.pdf>. Acesso em: 08 nov. 2018.

BRASIL, ANVISA. Agência Nacional de Vigilância Sanitária. Regulamento Técnico sobre Rotulagem Nutricional de Alimentos Embalados. Resolução RDC n 360, de 23 de dezembro de 2003. Diário Oficial da União. Brasília- DF, 2003. Disponível em: $<$ http://portal.anvisa.gov.br/documents/33880/2568070/res0360_23_12_2003.pdf/5d4fc71 3-9c66-4512-b3c1-afee57e7d9bc>. Acesso em: 10 nov. 2018.

BUTTRISS, J. L.; STOKES, C. S. Dietary fibre and health: an overview. Nutrition Bulletin, v. 23, n. 3, p. 186-200, 2008. Disponível em: <https://onlinelibrary.wiley.com/doi/full/10.1111 /j.1467-3010.2008.00705.x>. Acesso em: 11 nov. 2018. 
DANIEL, A. P; BOCHI, V. C.; STEFFENS, C.; SILVA, L. P.; EMANUELLI, T. Fracionamento a seco da farinha de aveia e modificação química da fração rica em amido. 2006. $8 \mathrm{f}$. Dissertação (Fracionamento a seco da farinha de aveia e modificação química da fração rica em amido. Programa de Pós-Graduação em Ciência e Tecnologia dos Alimentos) Centro de Ciências Rurais, Universidade Federal de Santa Maria, Santa Maria, 2006. 26(4). Disponível em: <http://www.scielo.br/pdf/cta/v26n4/33.pdf>. Acesso em: 10 nov. 2018.

DENG, J.; LIU, Q.; ZHANG, Q.; LIU, D.; FAN, D.; YANG, H. Comparative study on composition, physicochemical and antioxidant characteristics of different varieties of kiwifruits seed oil in China. Food Chem. V. 264, p. 411-418, out. 2018. Disponível em: <https://www.ncbi.nlm.nih.gov/pubmed/29853395>. Acesso em: 26 out. 2018.

EMBRAPA - Cultivo da melancia em Rondônia / Flávio de França Souza, Editor Técnico. Porto Velho: Embrapa Rondônia, 103 p., 2008.

EMBRAPA - Sistema de Produção de Melancia. Embrapa Semiárido. Sistemas de Produção, 6. Ago/2010. Disponível em: <https://sistemasdeproducao.cnptia.embrapa.br/ FontesHTML/Melancia/SistemaProducaoMelancia/socioeconomia.htm>. Acesso em: 08 nov. 2018.

FONSECA, H., GUTIERREZ, L. E. Composição em ácidos graxos de óleos vegetais e gorduras animais. In: Anais da E.S. A. Luiz de Queiroz, 1974. Composição em ácidos graxos de óleos vegetais e gorduras animais. São Paulo: 1974. p. 485-490. v. XXXI.

GARG, A. High-monounsaturated-fat diets for patients with diabetes mellitus: a metaanalysis. The Amer. J. of Clin. Nut., v. 67, n. 3, p. 577-582, mar. 1998. Disponível em: <https://www.ncbi.nlm.nih.gov/pubmed/9497173>. Acesso em: 10 nov. 2018.

GAULLIER, J. M.; HALSE, J.; HOYE, K.; KRISTIANSEN, K.; FAGERTUN, H; VIK, H.; GUDMUNDSEN, O. Conjugated linoleic acid supplementation for 1 y reduces body fat mass in healthy overweight humans. The Amer. J. of Clin. Nut., v. 79, n. 6, p. 1118-1125, jun. 2004. Disponível em: <https://academic.oup.com/ajcn/article/79/6/1118/4690231>. Acesso em: 08 nov. 2018. 
GUIMARÃES, R. R. Bolos simples elaborados com farinha de entrecasca de melancia (Citrullus vulgaris, sobral): avaliação química, física e sensorial. Ciênc. Tecnol. Aliment., v. 2, n. 30, p. 354-363, jun. 2010. Disponível em: <http://www.scielo.br/pdf/cta/v30n2/11.pdf> Acesso em: 16 set. 2018.

GUTKOSKI, L. C.; BONAMIGO, J. M.; TEIXEIRA, D. M. F.; PEDÓ, I.. Desenvolvimento de barras de cereais à base de aveia com alto teor de fibra alimentar. Ciên. Tecnol. Aliment., Campinas, v. 27, n. 2, p. 355-363, abr. 2007. Disponível em: <http://www.scielo.br/pdf/cta/ v27n2/24.pdf>. Acesso em: 08 nov. 2018.

JASMINE, Alimentos Ltda. Farinha de Chia: Informação nutricional. 1. 2017. Disponível em: <https://www.jasminealimentos.com/produtos/integral/farinha-de-chia-estabilizada/>. Acesso em: 8 nov. 2018

IAL. INSTITUTO ADOLFO LUTZ. Normas analíticas do Instituto Adolfo Lutz: métodos físico químicos para análise de alimentos. 4. ed. São Paulo: Instituto Adolfo Lutz. 1020p, 2008.

IBGE. Produção Agrícola Municipal: Tabela 1612 - Área plantada, área colhida, quantidade produzida, rendimento médio e valor da produção das lavouras temporárias. 1 . 2017. Disponível em: <https://sidra.ibge.gov.br/tabela/1612>. Acesso em: 16 set. 2018

MINISTÉRIO DA SAÚDE. Rotulagem nutricional obrigatória: manual de orientação às indústrias de Alimentos - 2º Versão / Agência Nacional de Vigilância Sanitária Universidade de Brasília - Brasília: Ministério da Saúde, Agência Nacional de Vigilância Sanitária / Universidade de Brasília, 2005. 44p.

MARTIN, C. A.; ALMEIDA, V. V.; RUIZ, M. R.; VISENTAINER, J. E. L; MATSHUSHITA M.; SOUZA, N. E.; VISENTAINER, J. V. Ácidos graxos poliinsaturados ômega-3 e ômega-6: importância e ocorrência em alimentos. Revista de Nutrição, Campinas, v. 19, n. 6, p. 761770, nov. 2006. Disponível em: <http://www.scielo.br/pdf/rn/v19n6/10.pdf>. Acesso em: 09 nov. 2018.

PRIM, M. B. S. Análise do desperdício de partes vegetais consumíveis. 2003. $117 \mathrm{f}$. 
Dissertação (Análise do desperdício de partes vegetais consumíveis. Programa de Pós Graduação em Engenharia de Produção) - Universidade Federal de Santa Catarina, Florianópolis, 2003. Disponível em: <https://core.ac.uk/download/pdf/30367658.pdf>. Acesso em: 10 nov. 2018

PUIG, E. I.; HAROS, M. La Chia en Europa: EI Nuevo Ingrediente en Productos de Panadería. Alimentaria, Lugo, v. 420, p. 73-77, 2011. Disponível em: <https://www.researchgate.net/publication/285785109_La_chia_em_Europa_El_nuevo_in grediente_en_productos_de_panaderia>. Acesso em: 25 nov. 2018

SELF NUTRITION DATA. Seeds, watermelon seed kernels, dried Nutrition Facts \& Calories: Seeds, watermelon seed kernels, dried. 1. 2018. Disponível em: <https://nutritiondata.self.com/facts/nut-and-seed-products/3147/2>. Acesso em: 10 nov. 2018.

TACO. Tabela brasileira de composição de alimentos. 4.ed. Campinas: NEPAUNICAMP, 2011

WHIGHAM, L. D.; WATRAS, A. C.; SCHOELLER, D. A. Efficacy of conjugated linoleic acid for reducing fat mass: a meta-analysis in humans. The American Journal of Clinical Nutrition, v. 85, n. 5, p. 1203-1211, maio. 2007. Disponível em: <https://www.ncbi.nlm.nih. gov/pubmed/17490954>. Acesso em: 09 nov. 2018. 